\title{
Adaptation of Web Service Composition Based on Workflow Patterns
}

\author{
Qiang $\mathrm{He}^{1,2}$, Jun Yan $^{3}$, Hai Jin ${ }^{1}$, and Yun Yang ${ }^{2}$ \\ ${ }^{1}$ School of Computer Science and Technology, \\ Huazhong University of Science and Technology, Wuhan, China 430074 \\ hjinahust.edu.cn \\ ${ }^{2}$ Faculty of Information and Communication Technologies, \\ Swinburne University of Technology, Melbourne, Australia 3122 \\ qhe@ict.swin.edu.au, yyang@swin.edu.au \\ ${ }^{3}$ School of Information Systems and Technology, \\ University of Wollongong, Wollongong, Australia 2522 \\ jyan@uow.edu.au
}

\begin{abstract}
Business processes consisting of component Web services are often executed in volatile environments where the quality of service parameters of the participating services might change during the execution of the business processes. Recently, research has been carried out on adapting composite Web service in volatile service-oriented computing environments. However, current approaches do not consider the internal logic of the business process and the impact of adaptation for a single service on the other component services. Other than quality of service parameters, effective adaptation requires specific information of the component services in terms of their position and interaction in the business process. The work reported in this paper is a first step in this direction. We present a novel approach to adaptation of Web service composition based on workflow patterns. This approach measures the value of changed information that updated services may potentially introduce in the business process. Experimental results show that our approach provides effective adaptation solutions by expanding the adaptation scope and considering the internal logic of business process.
\end{abstract}

Keywords: Adaptation, Business Process, Quality of Service, Adaptation, Service Level Agreement, Web Service Composition.

\section{Introduction}

In service-oriented computing (SOC), one of the most important functions is to create value-added services, i.e. service compositions, by composing existing services, namely component services. A composite service can be modelled as a business process with the internal logic between the component services captured using a business process modelling language tailored for Web services, e.g. BPEL [1, 12], WSCI [3] and BPSS [5]. The delivery of a composite service is achieved through the coordinated invocation of component services. 
Quality of service (QoS) control for service compositions is very difficult to manage due to the cross-organisation and location-distribution of Web services. To solve this problem, service level agreements (SLA) $[10,16]$, referring to mutually agreed understanding and expectation about service provision, can be established between service consumers and service providers. WS-Agreement $[2,13]$ is the de facto SLA specification standard for Web services. In service composition scenarios, QoS control is critical, as any failure to meet local requirements of the component services may result in exceptions of the composite service. In these scenarios, the service consumer will contract SLAs with the service providers over each individual component service. The aggregation of QoS of component services is supposed to meet the global requirements of the client. However, the SOC environment is always volatile in which contracted SLAs would potentially be violated due to the inherent unreliability of the underlying Internet and internal infrastructures of service providers. When a violation of SLA occurs, the corresponding component service needs to be recovered with satisfying QoS to meet the global business process requirements. During the recovery process, cost applies. Therefore, when recovering the failed service the service provider that brings the greatest estimated profit - estimated value minus cost - should be selected. The value of changed information (VOC) [6] is used to compute the tradeoff between the expected value and the cost from updating the service. The update is performed only when it is going to pay off. Here the VOC computation shares its concepts with the value of perfect information (VPI) [15], which also attempts to decide whether new information is necessary and useful to a particular process, as explained in [6]. Usually, "update" refers to changing QoS of the services. Since recovering the services also incurs cost while bringing benefit, VOC related mechanisms can also be applied to recover services. There are two typical methods to recover failed services in an executing business process instance: 1) renegotiate with the failed service provider; 2) replace the failed service provider with a new service provider. Both methods will incur recovery cost. Generally speaking, the recovery cost occurs upon querying the information, renegotiating and negotiating with service providers, and switching service providers. The optimal solution for service adaptation is to select the solution that brings the most tradeoff, i.e. VOC minus recovery cost. The computation of the VOC and recovery cost are domain-specific and thus out the scope of this paper.

So far, little efforts have been placed on the business process adaptation which considers the impact of the adaptation for one service on the other services. When service recovery is required, e.g. current service providers claim to be incapable of providing promised services or service providers fail to deliver results as specified in the SLA, it is sometimes essential to update a group of component services because otherwise the global QoS requirements of the business process cannot be met. For example, it takes a certain amount of time to recover the failed service, which, with the addition of pre-specified time consumption of the remaining unexecuted component services, will lead to violation of the global requirements of time consumption on the whole business process and thus requires adaptation of more unexecuted component services. Therefore, a comprehensive adaptation mechanism that involves recovering and updating services is imperative to the generation of optimal adaptation solution. Besides, adaptation is supposed to be confined within a certain scope, usually the smaller the better, to limit the impact of failed services on other services and the cost 
and complexity of adaptation. Hence, defining the scope and furthermore, the adaptation solution is considerably significant. Workflow patterns [17] define specific internal workflow logics - elicited from multiple homogeneous cases - and provide reusable models for developers to deliver solid, proper architecture solutions. In this paper, we discuss how to apply VOC to the generation of adaptation solutions in the scope of component services of the business process captured using pre-defined workflow patterns.

The rest of the paper is organised as follows. Section 2 introduces the major related work. Then, Section 3 analyses the requirements of adaptation in service composition scenarios by illustrating a motivating example. After that, Section 4 discusses the VOC computation based on workflow patterns followed by Section 5 which presents a method that utilises the approach presented in Section 4. Finally, Section 6 describes the experiments to demonstrate the effectiveness of the approach and Section 7 summarises the major contribution of this paper and outlines authors' future work.

\section{Related Work}

Recently, business process adaptation in dynamical and volatile environments has attracted increasing attention. Harney and Doshi [6] present a mechanism called VOC which computes the estimated value brought by the changes of the business process and compares the value to the cost required to make the changes. The update is performed only when it is expected to pay off. In [7], Harney and Doshi utilise service expiration times to reduce the computational overhead of adaptation. The improvement is based on the insight that service providers often keep the quality of their services at a certain level for a period of time. A new approach is proposed, namely VOC with expiration times $\left(\mathrm{VOC}^{\varepsilon}\right) . \mathrm{VOC}^{\varepsilon}$ manages to reduce the computational burden of adaptation. However, while considering adapting one individual service, the effect of VOC and $\mathrm{VOC}^{\varepsilon}$ is limited on just the one service without taking into account the global business process. In other words, VOC and $\mathrm{VOC}^{\varepsilon}$ facilitate only local adaptation solutions for business process which cannot guarantee the satisfaction of the global business process requirements.

Chafle et al. [4] introduce adaptation on different levels, including the instance level, logic level and physical level. Multiple backup workflows are prepared to substitute the failed components or workflows at any moment. By enabling this, workflow systems can adapt to environmental changes. Verma et al. [18] introduce a suite of stochastic optimisation based methods, including centralised and decentralised ones for adapting business process modelled as Markov decision processes. Exogenous events and inter-service constraints are both taken into account when performing the adaptation. Narendra et al. [14] use the aspect-oriented programming (AOP) technology to dictate modifications in component services in order to meet non-functional requirement changes in the composite service. None of the above approaches consider the cost that may occur in the adaptation of business processes and may generate worthless adaptation solutions which may bring cost more than profit. Our approach aims at addressing the above mentioned shortcomings. 


\section{A Motivating Example}

In order to illustrate the needs for and the complexity of service composition adaptation, in this section we present a motivating example.

This example business process involves a trading company, a manufacturer, a road transportation company and a shipping company. The trading company wants to purchase some goods which will be produced by the manufacturer, and then delivered by road transportation company A and shipping company A in sequence. The SLAs for the manufacturing, road transportation and shipping have been negotiated before services are provided, which collectively fulfil the global requirements. Suppose that the road transportation company A suddenly claims a delay and cannot provide the service within the timeframe as promised in the SLA, this may delay the whole process. Therefore, the business process must have adaptation capabilities to recover from this exceptional situation. In addition, it is possible that the schedule of shipping company A will also be disturbed. For example, the shipping company may specify the constraint such as "Shipping service will be completed with 10 days during May 1 to May 30." in SLA. Therefore, with the road transportation service rearranged, this constraint may no longer be held. Thus, the shipping service needs to be coordinated by either renegotiating over the shipping SLA between the trading company and shipping company A or selecting a new shipping company.

In the adaptation process, cost applies, including the service query cost and the negotiation cost. The trading company may recover the road transportation service by either renegotiating with road transportation company A or finding another service provider, say road transportation company B or C, to replace road transportation company A. To select from the candidate service providers for road transportation service, the trading company needs to estimate the value and cost that candidate service providers may bring. Meanwhile, the trading company needs to compute the probability that the recovery of road transportation service will lead to disturbance of the shipping service and the corresponding value and cost from adapting the shipping service. Based on the results, the trading company will decide how to recover the road transportation service, whether to update the shipping service and if so, how.

In this case, the optimal solution is not necessarily selecting the companies with the best quality but the one with the best tradeoff between the value and cost. This motivating example reveals three important factors for selecting the optimal adaptation solution. First, the trading company must estimate the tradeoff between the value and cost brought by the candidate service providers. Second, the trading company must also estimate the impact drawn from the recovered services on other services to see if they need to be updated. Third, the cost for rearranging other services must be considered.

\section{Adaptation for Composite Service Based on Workflow Patterns}

Web service composition can be modelled as executable process using Web service composition languages like BPEL and WSFL incorporating the concepts and mechanisms in the workflow community. Therefore, the logic in a composite service can be captured using workflow patterns. In this section, we consider the workflow patterns 
presented in [17], and discuss and analyse how the VOC mechanism can be extended based on these patterns to facilitate adaptation in Web service composition. The services discussed are generic and their states are recoverable.

\subsection{Sequence Pattern}

- Pattern 1 Sequence. A Sequence pattern describes the structure where a component service starts after the completion of another component service in the same process.

When service $A$ violates the SLA, there are two ways to recover it. The business process manager can either renegotiate with the current service provider to see if it can still provide service $A$ to meet the global business process requirements, or find a new service provider to replace it. Before performing the adaptation process, the VOC of each of the above measures needs to be computed.

Since the actual values of the updated QoS are not known until after querying the service providers, we average all the possible combination of the values of updated QoS of the service using current belief distributions which can be obtained from the pre-defined SLAs, previous interactions with the service providers or a third SLA profiling center [8]. For each candidate service provider for service $A$ (including the original service provider), an estimated value is computed. Formally,

$$
V\left(A \mid A^{\prime}\right)=\int_{\Omega_{A^{\prime}}} u(e, p) \cdot \operatorname{Pr}\left(E_{A^{\prime}}=e, P_{A^{\prime}}=p\right) d \Omega_{A^{\prime}}{ }^{1} .
$$

where $V\left(A \mid A^{\prime}\right)$ is the estimated value from recover service $A$ with service provider $A^{\prime}$, $u(e, p)$ is a utility function which computes the utility of a service based on the client's preferences for execution time, $e$, and price, $p, \operatorname{Pr}(E=e, P=p)$ denotes the belief distribution of the combination $(e, p)$ and $\left.\Omega_{A^{\prime}}=<\left(e_{1}, p_{1}\right),\left(e_{2}, p_{2}\right), \ldots,\left(e_{n}, p_{n}\right)\right\rangle_{A^{\prime}}$, represents the possible combinations of the values of execution time and price from the candidate service providers for service $A$. Here we take the originally contracted service provider as a candidate service provider if it is still potentially capable of providing the service with amended SLA.

Then the process manager computes the probability that service $B$ needs to be rearranged due to selecting service provider $A^{\prime}$ to recover service $A$. The probability, denoted as $P\left(A \mid A^{\prime} \rightarrow B\right)$, is affected by three main aspects:

1) the extra resource consumption caused by the adaptation of service $A$. For example, delay and increased price from adapting service $A$ will increase the need of updating service $B$;

2) the margins of the related terms in the SLA contracted for services $A$ and $B$. If the business process manager had succeeded in contracting an SLA with extra marginal violation tolerance, there would be a relatively low chance that service $B$ needs to be adapted; and

3 ) the estimated capability of candidate service providers for service $A$. If it is expected to seal a deal with a candidate service provider that is capable of providing service $A$ with better QoS, the need of updating service $B$ will decrease.

\footnotetext{
${ }^{1}$ In this paper we use execution time and price for the purpose of demonstration.
} 
In the sequence pattern, the formal computation of $P_{S e q}\left(A \mid A^{\prime} \rightarrow B\right)$ is:

$P_{\text {Seq }}\left(A \mid A^{\prime} \rightarrow B\right)=\frac{1}{\left|\Omega_{A^{\prime}}\right|} \cdot \int_{\Omega_{A^{\prime}}}$ isGlobal Re quirementViolated $(e, p) \cdot \operatorname{Pr}\left(E_{A^{\prime}}=e, P_{A^{\prime}}=p\right) d \Omega_{A^{\prime}}$.

where isGlobalRequirementViolated $(e, p)$ is a function that, given the execution time and price, returns 1 if the global business process requirements will be violated and 0 otherwise. This function involves QoS aggregation [9] and multiple criteria decision making (MCDM) [11] and is implemented application-specifically.

Then we formulate the VOC due to the service adaptation as:

$$
\begin{gathered}
V O C_{A\left|A^{\prime}+B\right| B^{\prime}}=V\left(A \mid A^{\prime}\right)+P\left(A \mid A^{\prime} \rightarrow B\right) \cdot V\left(B \mid B^{\prime}\right) \\
=\int_{\Omega_{A^{\prime}}} u(e, p) \cdot \operatorname{Pr}\left(E_{A^{\prime}}=e, P_{A^{\prime}}=p\right) d \Omega_{A^{\prime}} \\
+P_{S e q}\left(A \mid A^{\prime} \rightarrow B\right) \cdot \int_{\Omega_{B^{\prime}}} u(e, p) \cdot \operatorname{Pr}\left(E_{B^{\prime}}=e, P_{B^{\prime}}=p\right) d \Omega_{B^{\prime}} .
\end{gathered}
$$

where $V O C_{A\left|A^{\prime}+B\right| B^{\prime}}$ is the value brought from adapting service $A$ with service provid er $A^{\prime}$ and service $B$ with service provider $B^{\prime}$.

Since adapting services $A$ and $B$ may be expensive, the adaptation is performed only when it is expected to pay off. The adaptation cost, including querying information, renegotiating and negotiating with service providers, and switching service providers, must be lower than the corresponding VOC. Formally, the adaptation is performed when:

$$
\operatorname{VOC}_{A\left|A^{\prime}+B\right| B^{\prime}}>\operatorname{COST}\left(A\left|A^{\prime}+B\right| B^{\prime}\right) .
$$

where $\operatorname{COST}\left(A\left|A^{\prime}+B\right| B^{\prime}\right)$ is the adaptation cost from recovering service $A$ with service provider $A^{\prime}$ and service $B$ with service provider $B^{\prime}$. When there are more than one qualified combination of candidates for services $A$ and $B$, the one with the greatest profit, i.e. $V O C_{A\left|A^{\prime}+B\right| B^{\prime}}-\operatorname{COST}\left(A\left|A^{\prime}+B\right| B^{\prime}\right)$, is selected.

From formulas (1) - (4), we can observe that it is the combination of candidates for services $A$ and $B$ that determines the profit from the adaptation process. The business process manger needs to compute the VOC of all the possible combinations of candidates for services $A$ and $B$, which is computationally intensive if the number of candidates is large. Under this circumstance, the business process manger can select several candidates according to the ranking provided by an SLA profiling centre.

\subsection{Parallel Patterns}

Besides the sequence pattern, parallel is another major pattern in any model of business processes. The authors in [17] consider the parallel patterns in terms of (1) how the branches are picked, (2) how they are executed and (3) how they converge. In this section, we analyse the adaptation mechanisms for different types of parallel patterns considering the three aspects above. 
There are three typical split patterns that describe the logic of processes splitting and proceeding:

- Pattern 2 Parallel Split. A Parallel Split describes the structure where a single thread splits into multiple threads which can be executed in parallel. In this pattern, component services $A$ and $B$ will both be executed and can be executed simultaneously in any order.

- Pattern 3 Exclusive Choice. An Exclusive Choice pattern descries the structure where, based on a decision or process control data, only one selected branch is activated and executed.

- Pattern 4 Multi-Choice. A Multi-Choice pattern describes the structure where, based on a decision or process control data, a number of branches are chosen.

We must also consider how the branches will converge (if they will). There are five typical patterns that model the logic of the branches converging:

- Pattern 5 Synchronisation. A Synchronisation pattern describes the structure where multiple parallel branches converge into one single thread synchronised.

- Pattern 6 Simple Merge. A Simple Merge pattern describes the structure where more than one branches converge without synchronisation and only one of them has ever been executed.

- Pattern 7 Synchronising Merge. A Synchronising Merge pattern describes the structure where synchronising happens only when more than one branches are active (i.e. they are being executed).

- Pattern 8 Multi-Merge. A Multi-Merge pattern describes the structure where the branches converge without synchronisation and the service succeeding the mergence will be activated by the completion of every incoming branch.

- Pattern 9 Discriminator. A Discriminator pattern describes the structure where the subsequent service will be activated by the first and only the first completed branch. The remaining branches will be ignored.

The combination of the split patterns and converge patterns determines the adaptation solution when services need to be recovered. Starting with the simplest combination, i.e. Parallel Split + Synchronisation, we discuss the corresponding adaptation mechanisms.

\section{Parallel Split + Synchronisation}

In this pattern combination, there are more than one branches splitting at a certain point and then converge with synchronisation at the end of the completion of all the branches. Here we suppose there is only one service on each parallel branch. This assumption is realistic because if there are more than one services on any branch they can be considered as a composite service. When the service on one of the parallel branches needs to be recovered, the business process manager must compute the VOC of the service and select an appropriate service provider. Moreover, to guarantee global business process requirements satisfaction, the business process manager must also consider if it is necessary to update the services on other branches.

We consider the adaptation under two circumstances: 1) all the branches have not been activated and executed and the service provider for one of them claims to be unable to provide the service, or incapable of providing the service with promised 


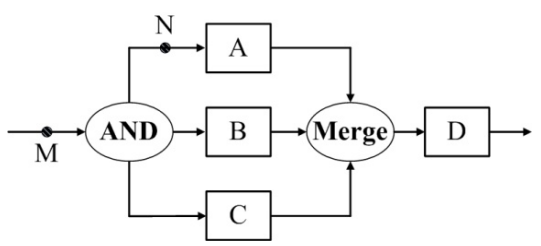

Fig. 1. Parallel Split + Synchronisation

quality. In this case, the adaptation happens before point $M$ in Figure 1 ; 2) all the branches have been activated and one of the service providers fails to deliver the required result. In this case, the adaptation happens after points like $\mathrm{N}$.

In case 1), the business process manger needs to estimate if the adaptation of failed service, i.e. service $A$ in Figure 1, will cause the delay of activation of service $D$ due to the time consumption of the adaptation process and the newly contracted SLA. If it is true, the business process manager can think about giving services $B$ and $C$ more time to complete because given more flexible time constraint, the business process manager may be able to renegotiate with service providers of $B$ and $C$ for a lower price or better QoS if the SLAs previously contracted for services $B$ and $C$ are renegotiable and modifiable.

For the pattern combination, Parallel Split + Synchronisation, the probabilities of additional execution time for services $B$ and $C$ caused by the adaptation of service $A$, assuming service $A$ is replaced by service $A^{\prime}$, are computed as:

$$
\begin{aligned}
& P_{P S P}\left(A \mid A^{\prime} \rightarrow B\right)=\frac{1}{\left|E_{A^{\prime}}\right|} \cdot \int_{E_{A^{\prime}}} \cdot \operatorname{Pr}\left(E_{A^{\prime}}=e\right) \cdot i \operatorname{isigger}\left(e, e_{B}\right) d E_{A^{\prime}} . \\
& P_{P S P}\left(A \mid A^{\prime} \rightarrow C\right)=\frac{1}{\left|E_{A^{\prime}}\right|} \cdot \int_{E_{A^{\prime}}} \cdot \operatorname{Pr}\left(E_{A^{\prime}}=e\right) \cdot \operatorname{isBigger}\left(e, e_{C}\right) d E_{A^{\prime}} .
\end{aligned}
$$

where $e_{B}$ and $e_{C}$ are the execution times for services $B$ and $C,\left|E_{A^{\prime}}\right|$ is the modulo of $E_{A^{\prime}}$, and $\operatorname{isBigger}(x, y)$ is a function returns 1 when $\mathrm{x}$ is bigger than $\mathrm{y}$ and 0 otherwise. Then the VOC of different adaptation strategies, involving services $A, B$ and $C$, can be computed as:

$$
V O C_{P S P}^{A\left|A^{\prime}+B\right| B^{\prime}+C \mid C^{\prime}}=V\left(A \mid A^{\prime}\right)+P\left(A \mid A^{\prime} \rightarrow B\right) \cdot V\left(B \mid B^{\prime}\right)+P\left(A \mid A^{\prime} \rightarrow C\right) \cdot V\left(C \mid C^{\prime}\right) .
$$

In case 2), when the exception is detected on service $A$, services on other branches, i.e. services $B$ and $C$, have already been activated. When the adaptation for service $A$ is being performed, services $B$ and $C$ are already under execution. Therefore, the estimated time consumption from adapting service $A$, must be involved in the computation of $P\left(A \mid A^{\prime} \rightarrow B\right)$ and $P\left(A \mid A^{\prime} \rightarrow C\right)$ :

$$
\begin{aligned}
& P_{P S P}\left(A \mid A^{\prime} \rightarrow B\right)=\frac{1}{\left|E_{A^{\prime}}\right|} \cdot \int_{E_{A^{\prime}}} \cdot \operatorname{Pr}\left(E_{A^{\prime}}=e, E_{\text {adap }}=e_{\text {adap }}\right) \cdot \operatorname{isBigger}\left(e+e_{A D A P}, e_{B}\right) d E_{A^{\prime}} . \\
& P_{P S P}\left(A \mid A^{\prime} \rightarrow C\right)=\frac{1}{\left|E_{A^{\prime}}\right|} \cdot \int_{E_{A^{\prime}}} \cdot \operatorname{Pr}\left(E_{A^{\prime}}=e, E_{\text {adap }}=e_{\text {adap }}\right) \cdot \operatorname{isBigger}\left(e+e_{A D A P}, e_{C}\right) d E_{A^{\prime}} .
\end{aligned}
$$

where $e_{A D A P}$ is the estimated time to adapt service $A$. 
Then the VOC of different adaptation solutions can be computed using formula (7) with $V\left(A \mid A^{\prime}\right), V\left(B \mid B^{\prime}\right)$ and $V\left(C \mid C^{\prime}\right)$ computed similarly as formula (1).

\section{Parallel Split + Multi-Merge}

In a Multi-Merge pattern, the service succeeding the mergence will be executed every time an incoming branch completes. Most of the workflow products, e.g. Eastman, Verve Workflow and Forte Conductor, implement the Multi-Merge pattern by replicating the service(s) succeeding the mergence (see Figure 2 for a simple example). And the replicated services will be made sequential to the services on each of the original branches, generating several independent sequence structures. Actually, at runtime, no Multi-Merge structures will be found. Therefore, VOC computation for Sequence pattern will be applied to the created Sequence structures in adaptation.

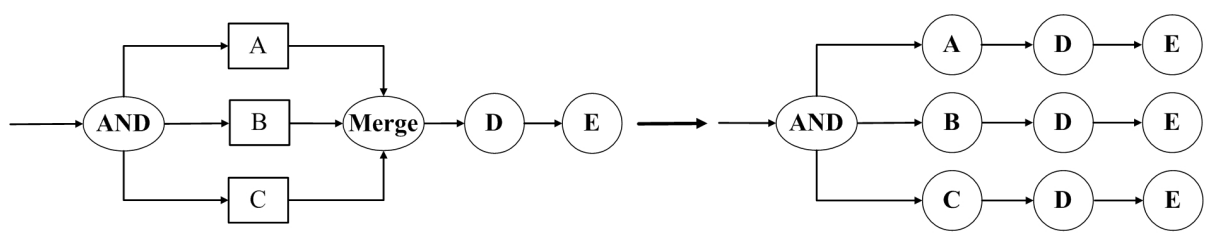

Fig. 2. Implementation of Multi-Merge pattern

\section{Parallel Split + Discriminator}

In a Discriminator pattern, the service succeeding the mergence waits for the first completed incoming branch and ignores the rest. In other words, the succeeding service will be activated only once when one of the incoming branches is firstly completed. We now discuss how to adapt the parallel services in terms of VOC.

In this pattern combination the fastest branch (the one with the shortest execution time) determines the start time of the service succeeding the mergence. Once the succeeding service is activated, the uncompleted branches will be ignored. In fact, Discriminator pattern is not found often in business processes where SLA is enabled because generally the execution time of the services will be specified in the SLA and hence it can be estimated that which branch is likely to complete first and which branches will be ignored. However, there is one exception: the business process manager wants to hedge the risk of delay caused by service provider failing to deliver expected result. In this case, by employing the Discriminator pattern, when a branch is broken, other branches can still deliver expected result in a relatively tolerant period of time. Intuitively, the most effective way to hedge the risk is to allocate low execution time for individual branches while obtaining a high successful global execution rate of the branches. It is also the major objective of adapting the branches when a branch is broken. Since the services on different branches are functionally equivalent, they share a group of candidate service providers and a utility function. For the demonstration purpose, we use the example in Figure 3. 


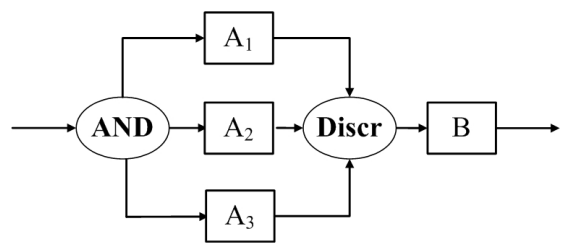

Fig. 3. Parallel Split + Discriminator

First, the normalised successful execution rate weights, $w_{1}, w_{2}$ and $w_{3}$, are assigned to the candidate service providers. The success rate weights range from 0 to 1 , representing how much trustworthy the service providers are based on their historical performance. Service providers with better historical performance, i.e. higher successful rate, will be assigned with higher weights. The historical performance of service providers may be provided by the service providers through pre-defined SLAs or they could be learnt from previous interactions with the service providers.

Second, the VOC can be computed as:

$$
\begin{gathered}
\operatorname{VOC}_{P S D}^{A\left|A^{\prime}+B\right| B^{\prime}+C \mid C^{\prime}}=\int_{\Omega_{\left(A^{\prime} ; B^{\prime} C^{\prime}\right)}} w_{l} \cdot u\left(e_{A^{\prime}}, p_{A^{\prime}}\right) \cdot \operatorname{Pr}\left(E_{A^{\prime}}=e_{A^{\prime}}, P_{A^{\prime}}=p_{A^{\prime}}\right) \\
+w_{2} \cdot u\left(e_{B^{\prime}}, p_{B^{\prime}}\right) \cdot \operatorname{Pr}\left(E_{B^{\prime}}=e_{B^{\prime}}, P_{B^{\prime}}=p_{B^{\prime}}\right)+w_{3} \cdot u\left(e_{C^{\prime}}, p_{C^{\prime}}\right) \cdot \operatorname{Pr}\left(E_{C^{\prime}}=e_{C^{\prime}}, P_{C^{\prime}}=p_{C^{\prime}}\right) d \Omega_{\left(A^{\prime}, B^{\prime}, C^{\prime}\right)} .
\end{gathered}
$$

Based on formula (10), the combination of candidate providers that maximises $V O C_{P S D}^{A\left|A^{\prime}+B\right| B^{\prime}+C \mid C^{\prime}}-\operatorname{Cost}\left(A \mid A^{\prime}\right)-\operatorname{Cost}\left(B \mid B^{\prime}\right)-\operatorname{Cost}\left(C \mid C^{\prime}\right)$ will be selected.

Sometimes which branches will be executed is dependent on runtime decision making. Pattern 3 Exclusive Choice and pattern 4 Multi-Choice describe the two different situations in this category. In pattern 3 Exclusive Choice, only one branch will be chosen and executed in a running process instance and it leads to a Simple Merge. That makes the branches uninfluential on one another. Therefore, when one branch needs to be recovered, other branches do not need to be considered. Yet the VOC mechanism can still be applied here because, the broken branch, together with the service succeeding the mergence, can be seen as a Sequence pattern. In pattern 4 Multi-Choice, multiple branches will be chosen for execution. Therefore, the business process manager needs to consider the other branches when trying to recover a branch. Next we discuss the pattern combinations involving the Multi-Choice pattern.

\section{Multi-Choice + Synchronizing Merge}

In this pattern combination, multiple branches will be chosen for execution and they will be synchronised when they merge. The way the branches merge is similar to pattern 5 Synchronisation. The difference is that not all the incoming branches will be activated for every running instance. If one of the branches is broken and needs to be recovered, the business process manager needs to estimate the probabilities of other branches being activated and whether they can benefit from updating.

Generally, there is no way to ascertain which branches will be activated for a specific running instance until the dynamic decision is made. However, the business process manager can estimate the probability that a branch will be activated based on the historical performance of the branches. For example, if a branch was executed 80 times out of the last 100 business process instances, we consider the branch will be selected with 
a probability of $80 \%$. The probability of being selected for each branch can be normalised as weights, $s_{1}, s_{2}, \ldots s_{n}$, ranging between 0 and 1 representing how important the branches based on the probability they will be selected for execution. Take the business process in Figure 1 as an example, with Parallel Split replaced with Multi-Choice. Based on this assumption the computation of the VOC for the adaptation solution can be formalised as:

$$
\begin{gathered}
V O C_{M C S M}^{A\left|A^{\prime}+B\right| B^{\prime}+C \mid C^{\prime}}= \\
s_{1} \cdot V\left(A \mid A^{\prime}\right)+s_{2} \cdot P\left(A \mid A^{\prime} \rightarrow B\right) \cdot V\left(B \mid B^{\prime}\right)+s_{3} \cdot P\left(A \mid A^{\prime} \rightarrow C\right) \cdot V\left(C \mid C^{\prime}\right) .
\end{gathered}
$$

where $V\left(A \mid A^{\prime}\right), V\left(B \mid B^{\prime}\right)$ and $V\left(C \mid C^{\prime}\right)$ are computed similarly to formula (1), $P_{M C S M}\left(A \mid A^{\prime} \rightarrow B\right)$ and $P_{M C S M}\left(A \mid A^{\prime} \rightarrow C\right)$ are computed similarly to formulas (5) and (6).

\section{Multi-Choice + Multi-Merge}

Similar to the Parallel Split + Multi-Merge pattern combination, in Multi-Choice + Multi-Merge pattern combination the branches will be transformed into several independent sequence structures before being executed. Thus, VOC computation for $\mathrm{Se}$ quence pattern will be applied when necessary.

\section{Multi-Choice + Discriminator}

In this pattern combination, a number of branches are selected and executed in parallel based on a decision dynamically made. The first branch that completes will trigger the service succeeding the mergence and after that other branches will be ignored. As discussed before, the aim of employing the Discriminator pattern is to achieve relatively tolerant execution time when service failure happens. Therefore, in adaptation solution determination, the branches that have higher successful execution rates and higher probabilities of being selected should be given higher preference. Here we adopt the weights used before: $w$, representing the successful execution rate, and $s$, representing the probability of branches being selected. Again, for three parallel branches, the formalised VOC computation is:

$$
\begin{aligned}
& V O C_{M C D}^{A\left|A^{\prime}+B\right| B^{\prime}+C \mid C^{\prime}}=\int_{\left.\Omega_{A^{\prime}, B^{\prime} C^{\prime}}\right)} s_{I} \cdot w_{1} \cdot u\left(e_{A^{\prime}}, p_{A^{\prime}}\right) \cdot \operatorname{Pr}\left(E_{A^{\prime}}=e_{A^{\prime}}, P_{A^{\prime}}=p_{A^{\prime}}\right) \\
& +s_{2} \cdot w_{2} \cdot u\left(e_{B^{\prime}}, p_{B^{\prime}}\right) \cdot \operatorname{Pr}\left(E_{B^{\prime}}=e_{B^{\prime}}, P_{B^{\prime}}=p_{B^{\prime}}\right) \\
& +s_{3} \cdot w_{3} \cdot u\left(e_{C^{\prime}}, p_{C^{\prime}}\right) \cdot \operatorname{Pr}\left(E_{C^{\prime}}=e_{C^{\prime}}, P_{C^{\prime}}=p_{C^{\prime}}\right) d \Omega_{\left(A^{\prime} B^{\prime}, C^{\prime}\right)} \cdot
\end{aligned}
$$

\subsection{Other Patterns}

In addition to the nine patterns addressed so far, there are other eleven patterns represented in [17] not mentioned. Some of them are used to describe the global properties and special activities of the business processes, including Arbitrary Cycles, Implicit Termination, Multiple Instance, Cancel Activity and Cancel Case. Another two patterns, Deferred Choice and Milestone, are used to specify the triggering condition of services based on decision dynamically made. The last pattern, Interleaved Parallel Routing, describes a set of services that are executed one by one in an arbitrary order decided at runtime. However, our work, VOC based on workflow patterns, is dedicated to analysing adaptation solution for business process based on specific influence 
between services in a confined recovery scope described using workflow patterns. The above eleven patterns do not serve the goal and thus are excluded in discussion from this paper.

\section{Adaptation Method}

Figure 4 shows the pseudo code for adapting the business process using the mechanism presented in Section 4. The algorithm takes one input - the service that requires recovery, denoted as $S_{0}$. The algorithm starts with identifying the pattern that $S_{0}$ belongs to (line 3). After the pattern identification, the VOC of different adaptation solutions is computed (line 7 for Sequence pattern and line 18 for Parallel pattern). The adaptation solution that is estimated to bring the most profit will be performed (lines 9 and 20). If the adaptation solution within the current scope specified with workflow patterns is not satisfactory, the component services in the current scope will be considered as a composite service (lines 12-13 and lines 23-24) and adaptation will be performed in a larger scope. The algorithm returns true if the business process is successfully adapted and false if all the unexecuted component services have been taken into account and still no satisfactory adaptation solution found.

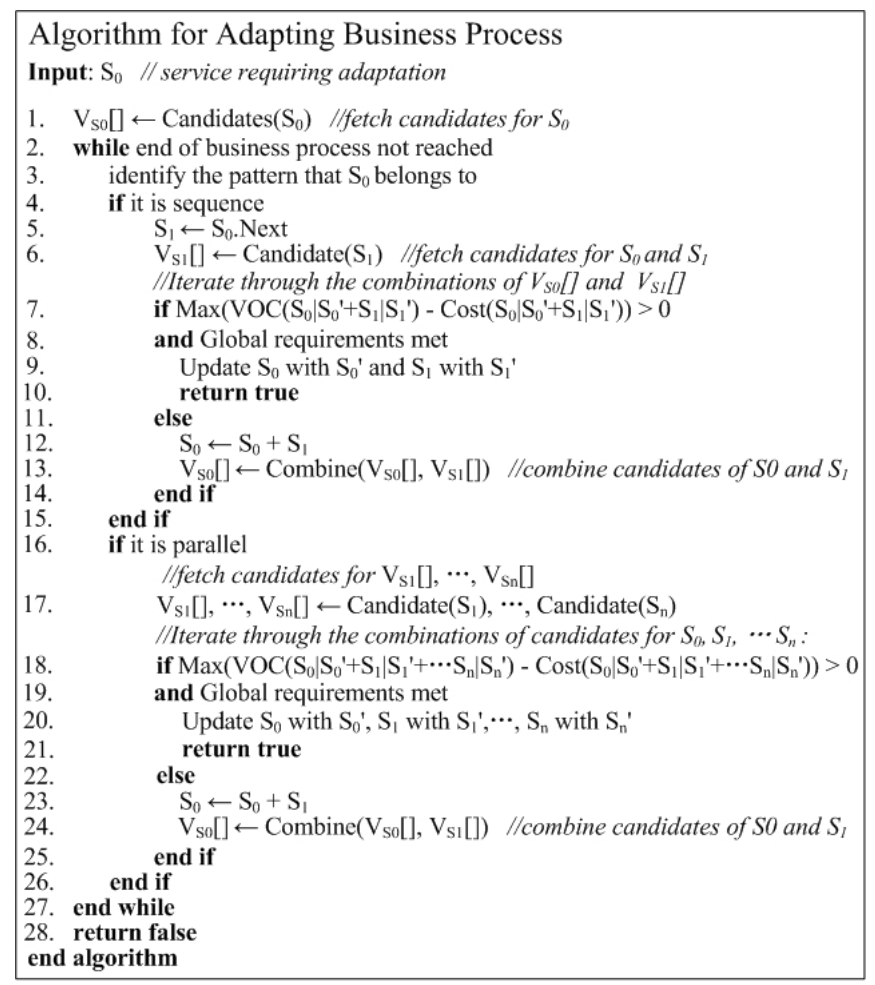

Fig. 4. Pseudo code for adapting a business process 


\section{Experimental Evaluation}

To evaluate the performance of our workflow based VOC approach for adapting business processes, we conducted experiments in a simulated volatile environment. The experimental evaluation is aimed at showing that our approach is effective in guaranteeing the satisfaction of the global business process requirements.

We utilised the goods purchase example presented in Section 3 for evaluation. This example is compliant with the Sequence pattern. Due to space limit, the results for other patterns are not presented. We evaluated the satisfaction rate of the global business process requirements with our adaptation approach enabled which considers both recovering the road transportation service and updating the shipping service. Considering that in different situations the difficulty levels of recovering and updating services might vary significantly, we model the road transportation companies' distribution and the shipping companies' distribution over their service satisfaction rates at two difficulty levels, i.e. easy and difficult, using beta distribution functions presented in Figure 5. Intuitively, service providers expose relatively high and low service satisfaction rates in respective easy and difficult situations.

We ran 1,000 independent business process instances for each experiment within a simulated volatile environment. Since the difficulty of adapting two sequential services might vary in different situations, we conducted comprehensive experiments with all

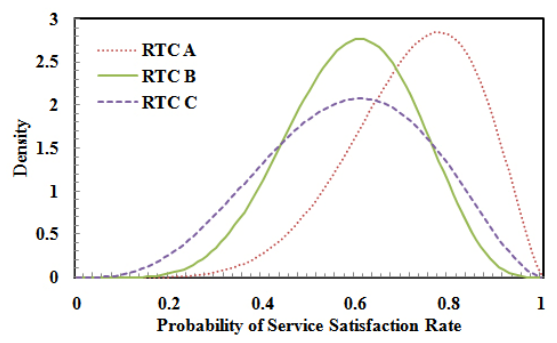

(a) Easy service satisfaction rates of road transportation companies

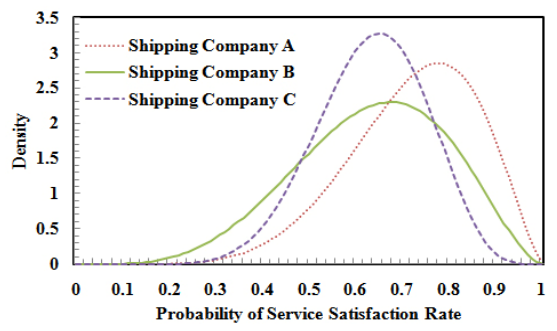

(c) Easy service satisfaction rates of shipping companies

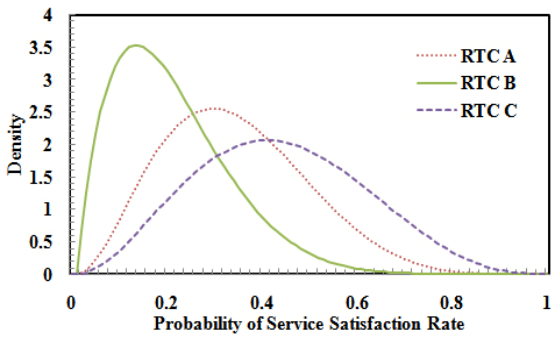

(b) Difficult service satisfaction rates of road transportation companies

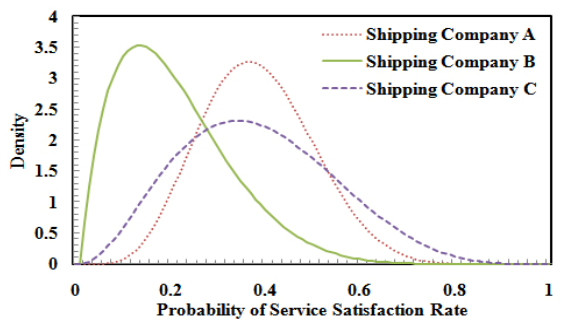

(d) Difficult service satisfaction rates of shipping companies

Fig. 5. Probability density functions 


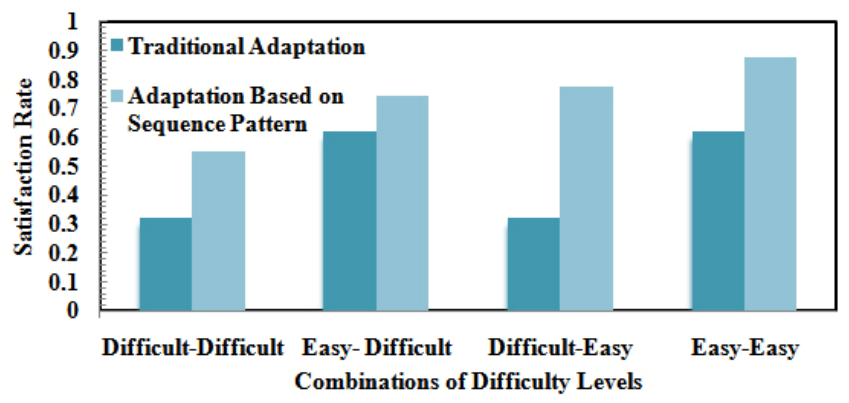

Fig. 6. Comparison between traditional adaptation and our adaptation for Sequence pattern

the four combinations of difficulty levels. We measured the satisfaction rate of the global requirements by calculating the successful cases, i.e. cases where global requirements can be met after the adaptation, out of the overall cases.

Figure 6 compares the satisfaction rates of global business process requirements in different situations with and without our approach enabled. The results demonstrate that in all situations our adaptation approach provide a more effective solution to the problem of global business process requirements satisfaction. In situations with different combinations of difficulty levels, including difficult-difficult, easy-difficult, difficult-easy and easy-easy, our approach provides an increment of $22 \%, 12 \%, 46 \%$ and $25 \%$ respectively in the satisfaction rates of global business process requirements.

\section{Conclusion and Future Work}

In open SOC environments, services can be volatile. Due to the inherent unreliability of the underlying Internet and internal infrastructures of service providers, SLA violation might happen. In service composition scenarios recovering just the failed service might not satisfy the global requirements of the composite service. Hence, business process adaptation needs to consider updating a certain scope of component services while recovering the failed one. Therefore, to determine the adaptation solution, we need to identify the adaptation scope and analyse the profit from different adaptation solutions. In this paper, we have discussed how the value of changed information (VOC) is extended and applied to business process adaptation based on workflow patterns. Specifically, we have analysed and presented how to compute VOC for sequence and different parallel pattern scenarios. When the adaptation is expected to pay off, it is performed within a certain scope defined by workflow patterns. In doing so, the business process adaptation can deliver satisfactory results while being kept within a reasonable scope. The experimental results show that our approach can significantly improve the satisfaction rates of global business process requirements in different situations.

In the future, we will apply the workflow-pattern-based VOC computation mechanism to our experimental prototype to test and analyse the performance of our approach. We will also attempt to improve the accuracy of VOC computation by utilising SLA profiling centre to provide historical and real-time performance of service providers. 
Acknowledgments. This work is partly funded by the Australian Research Council Discovery Project Scheme under grant No. DP0663841, National Science Foundation of China under grant No.90412010 and ChinaGrid project from Ministry of Education of China.

\section{References}

1. Andrews, T., Curbera, F., Dholakia, H., Goland, Y., Klein, J., Leymann, F., Liu, K., Roller, D., Smith, D., Thatte, S., Trickovic, I., Weerawarana, S.: Business Process Execution Language for Web Services Version 1.1 (2003),

http: / / download. boulder.ibm.com/ibmal/pub/software/dw/specs / ws-bpel/ws-bpel.pdf

2. Andrieux, A., Czajkowski, K., Dan, A., Keahey, K., Ludwig, H., Toshiyuki, N., Pruyne, J., Rofrano, J., Tuecke, S., Xu, M.: Web Services Agreement Specification (WS-Agreement): World-Wide-Web Consortium, W3C (2007),

http: / / www.ogf .org/documents/GFD.107.pdf

3. Arkin, A., Askary, S., Fordin, S., Jekeli, W., Kawaguchi, K., Orchard, D., Pogliani, S., Riemer, K., Struble, S., Takacsi-Nagy, P., Trickovic, I., Zimek, S.: Web Service Choreography Interface (WSCI) 1.0: World Wide Web Consortium, W3C (2002), http: // www.w3.org/TR/wsci/

4. Chafle, G., Dasgupta, K., Kumar, A., Mittal, S., Srivastava, B.: Adaptation in Web Service Composition and Execution. In: IEEE International Conference on Web Services, pp. 549-557. IEEE Computer Society, Chicago (2006)

5. Clark, J., Casanave, C., Kanaskie, K., Harvey, B., Clark, J., Smith, N., Yunker, J., Riemer, K.: ebXML Business Process Specification Schema Version 1.01: OASIS (2001), http: / / www . ebxml .org/specs / ebBPSS . pdf

6. Harney, J., Doshi, P.: Adaptive Web Processes Using Value of Changed Information. In: 4th International Conference on Service-Oriented Computing, pp. 179-190. Springer, Chicago (2006)

7. Harney, J., Doshi, P.: Speeding Up Adaptation of Web Service Compositions Using Expiration Times. In: 16th International Conference on World Wide Web, pp. 1023-1032. ACM, Banff (2007)

8. He, Q., Yan, J., Kowalczyk, R., Jin, H., Yang, Y.: Lifetime Service Level Agreement Management with Autonomous Agents for Services Provision Information Sciences (to appear, 2008)

9. Hwang, S.-Y., Wang, H., Tang, J., Srivastava, J.: A Probabilistic Approach to Modeling and Estimating the QoS of Web-Services-Based Workflows. Information Sciences 177(23), 5484-5503 (2007)

10. Jin, L.-J., Machiraju, V., Sahai, A.: Analysis on Service Level Agreement of Web Services. Technical Report, HP Laboratories (2002), http://www.hpl.hp.co.uk/ techreports / 2002/HPL-2002-180.pdf

11. Köksalan, M., Zionts, S.: Multiple Criteria Decision Making in the New Millennium. Springer, Heidelberg (2001)

12. Khalaf, R., Mukhi, N., Weerawarana, S.: Service-Oriented Composition in BPEL4WS. In: 12th International World Wide Web Conference (Alternate Paper Tracks), Budapest, Hungary (2003) 
13. Ludwig, H., Dan, A., Kearney, R.: Cremona: An Architecture and Library for Creation and Monitoring of WS-Agreements. In: 2nd International Conference on Service Oriented Computing, New York, USA, pp. 65-74 (2004)

14. Narendra, N.C., Ponnalagu, K., Krishnamurthy, J., Ramkumar, R.: Run-Time Adaptation of Non-functional Properties of Composite Web Services Using Aspect-Oriented Programming. In: 5th International Conference on Service-Oriented Computing, pp. 546-557. Springer, Vienna (2007)

15. Russell, S., Norvig, P.: Artificial Intelligence: A Modern Approach, 2nd edn. Prentice-Hall, Englewood Cliffs (2003)

16. Sturm, R., Morris, W., Hander, M.: Foundations of Service Level Management. SAMS (2000)

17. van der Aalst, W.M.P., ter Hofstede, A.H.M., Kiepuszewski, B.P.B.A.: Workflow Patterns. Distributed and Parallel Databases 14(1), 5-51 (2003)

18. Verma, K., Doshi, P., Gomadam, K., Miller, J.A., Sheth, A.P.: Optimal Adaptation in Web Processes with Coordination Constraints. In: IEEE International Conference on Web Services, pp. 257-264. IEEE Computer Society, Chicago (2006) 